\title{
Características da adoção de métodos, técnicas e ferramentas para inovação em organizações catarinenses
}

\author{
Gustavo Tomaz Buchele Mestre em Engenharia e Gestão do Conhecimento - Universidade Federal de Santa Catarina, Brasil - gustavotb.adm@gmail.com \\ Pierry Teza Mestre em Engenharia de Produção - Instituto Federal de Santa Catarina, Brasil - pierryteza@gmail.com \\ João Artur de Souza Doutor em Engenharia de Produção - Universidade Federal de Santa Catarina, Brasil - jartur@gmail.com \\ Gertrudes Aparecida Dandolini Doutora em Engenharia de Produção - Universidade Federal de Santa Catarina, Brasil - ggtude@gmail.com
}

\begin{abstract}
Resumo
A inovação tem sido considerada como elemento essencial não somente para o sucesso, mas para a sobrevivência das empresas a partir da criação de vantagem competitiva de longo prazo. Entretanto, esse processo ainda representa grandes desafios às organizações, sendo a adoção de Métodos, Técnicas e Ferramentas para Inovação um meio para estimulá-lo e apoiá-lo, aumentando suas chances de sucesso. Dessa forma, o objetivo deste artigo é descrever as características da adoção de Métodos, Técnicas e Ferramentas para Inovação em organizações catarinenses. Para isso, a pesquisa foi conduzida por uma abordagem quantitativa com coleta de dados realizada por meio de um levantamento (survey) em organizações catarinenses utilizando questionários fechados na internet. Como principais resultados verificou-se que são conhecidos muitos MTF-I, porém poucos são utilizados. Além disso, observou-se, assim como na literatura, que eles são mais frequentemente utilizados no front end da inovação. Foi possível identificar que a adoção de MTF-I é importante para aumentar a competitividade e que estes são utilizados principalmente para resolver problemas nas organizações. Nesse sentido, eles apresentaram alta satisfação após seu uso. A presente pesquisa contribui empiricamente com o tema, mesmo quando seus resultados não podem ser generalizados a todas as organizações catarinenses. Entretanto, tais resultados podem servir de ponto de partida para pesquisas futuras acerca do tema.
\end{abstract}

Palavras-chave: inovação; métodos, técnicas e ferramentas para inovação; MTF-l; organizações catarinenses; survey.

\section{Characteristics of adoption of methods, techniques and tools for innovation in organizations in Santa Catarina}

\begin{abstract}
Innovation has been considered an essential element not only for success, but for the survival of businesses by creating long-term competitive advantage. However, this process still poses major challenges to organizations, and the adoption of Methods, Techniques and Tools for Innovation is considered as means to stimulate it and support it, increasing its chances of success. Thus, the purpose of this article is to describe the characteristics of adoption of Methods, Techniques and Tools for Innovation in organizations in Santa Catarina. For this, the research was conducted following a quantitative approach with data collection conducted through a survey in organizations in Santa Catarina using questionnaires on the Internet. The main results found that many MTF-I are known but few are used. Furthermore, it was observed, as well as in the literature, they are most often used in the front end of innovation. It was possible to identify that the MTF-I adoption is important to increase the competitiveness and they are mainly used to solve problems in organizations. In this sense, they showed high level of satisfaction after being used. This study contributes empirically to the subject, even when the results can not be generalized to all organizations in Santa Catarina. However, these results can serve as a starting point for further research on the subject.
\end{abstract}

Keywords: innovation; methods, techniques and tools for innovation; MTF-l; organizations from Santa Catarina; survey. 


\section{INTRODUÇÃO}

Em um ambiente econômico competitivo e desafiador, a inovação se torna essencial não somente para o sucesso, mas para a sobrevivência das empresas (Nicholas et al., 2015). De acordo com Baregheh et al. (2009), a inovação é definida como um processo com diversas etapas em que as ideias são transformadas em produtos, serviços ou processos, novos/melhorados, com a finalidade de avançar, competir e diferenciar-se com sucesso em seu mercado. Tal processo, é vital para a vantagem competitiva de longo prazo das empresas. No entanto, motivar e estimular esse processo continua a ser um desafio para a maioria delas (Tian; Wang, 2014). Essa dificuldade se agrava devido à necessidade de rápidas tomadas de decisões de gestores frente a grande quantidade de informações disponíveis (Vaseashta, 2014).

Nesse contexto, uma dimensão de decisões importantes a serem tomadas em relação ao processo de inovação se refere a quais abordagens utilizar ao longo do processo. Essas abordagens suportam o entendimento, análise, decisão e ação ao longo do processo de inovação (Phaal et al., 2012). Entre essas abordagens, chamadas de Métodos Técnicas e Ferramentas para Inovação (MTF-Is) (Buchele et al., 2015) incluem brainstorming, análise morfológica, grupo focal, teste de conceito, cenários, retorno sobre o investimento (Nijssen; Lieshout, 1995; D'Alvano; Hidalgo, 2012), entre outros. O termo MTF-I é utilizado, uma vez que a literatura demonstra uma confusão da terminologia relacionada ao tema (Phaal et al., 2012) e poucos trabalhos fazem distinção entre eles (por exemplo, Shehabuddeen et al., 1999).

Diante disso, considera-se que um MTF-I pode ser um meio (ferramenta, documento, framework, procedimento, sistema, técnica ou método) que possibilita a organização a alcançar ou clarificar um objetivo (Brady et al., 1997). Na mesma acepção, Tidd e Thuriaux-Alemán (2016) utilizam o termo 'práticas de gestão da inovação' para se referir a um meio para modificar e aplicar a pesquisa em inovação e práticas de gestão. Os MTFIs, segundo lgartua et al. (2010), podem ajudar a gerir a inovação, auxiliando as organizações a se adaptarem às novas circunstâncias e enfrentarem os desafios de mercado de uma forma sistemática. Além disso, são fundamentais para aumentar a competitividade das organizações (Hidalgo; Albors, 2008), embora não possam garantir o sucesso.

Tendo em vista que foram identificados poucos trabalhos sobre a temática, verifica-se a necessidade de maiores aprofundamentos, especialmente por meio de trabalhos empíricos (Buchele, 2015). Nesta perspectiva, estabeleceu-se a seguinte pergunta de pesquisa: quais as principais características da adoção de métodos, técnicas e ferramentas para inovação em organizações catarinenses? Dessa forma, o objetivo deste estudo é descrever as principais características da adoção de MTF-Is a partir de um levantamento realizado em empresas catarinenses.

O termo "adoção" é bastante recorrente na literatura sobre MTF-I, assim como o termo "difusão". Ambos estão bastante relacionados, porém o primeiro se refere a decisão de uma empresa em utilizar ou não determinado MTF-I, já o segundo se refere ao número cumulativo de empresas que adotam um MTF-I ao longo do tempo (Chai; Xin, 2006). Embora haja essa relação, o foco deste trabalho é analisar a adoção, mesmo que aspectos sobre a difusão possam surgir de forma subjacente. Este artigo está estruturado em cinco seções. Esta primeira corresponde a introdução, na seção dois são apresentados os preceitos teóricos do trabalho, na terceira, os procedimentos metodológicos, enquanto que na quarta os resultados do levantamento realizado. Por fim, na quinta e última seção apresenta-se as considerações finais, bem como propostas para pesquisas futuras e as limitações deste trabalho.

\section{FUNDAMENTAÇÃO TEÓRICA}

Apresenta-se neste capítulo, inicialmente uma introdução acerca da inovação em Santa Catarina e em seguida os aspectos que fundamentam a temática Métodos, Técnicas e Ferramentas para Inovação.

\subsection{A inovação em Santa Catarina}

O Estado de Santa Catarina tem demonstrado um expressivo crescimento econômico. De acordo com o Ranking de Gestão e Competitividade dos Estados Brasileiros, levantamento realizado pelo grupo inglês Economist, em 2014 Santa Catarina se encontrava na quarta posição em relação aos indicadores de inovação. Esse 
crescimento parte de uma economia competitiva, a qual se destaca pela diversificação, pelo empreendedorismo, pelas condições sociais e institucionais, e pela valorização do capital humano (SEBRAE, 2010). Além disso, o Estado tem atraído investimentos por meio de ações de incentivos do Governo. Uma delas, o incentivo à inovação, tem ganhado destaque nos últimos anos, pois há esforços governamentais para a implantação de novos polos de inovação pelo Estado (SEBRAE, 2014).

Quanto as tendências para o Estado, o setor de tecnologia da informação e comunicação, o qual faz parte de um segmento produtivo intensivo em profissionais qualificados (2010), tem ganhado destaque demonstrando perspectivas de forte crescimento (SEBRAE, 2014). Nesse sentido, ações como os sistemas de Ciência, Tecnologia e Inovação, e as iniciativas e incentivos de políticas públicas, como por exemplo, a Lei de Incentivo à Inovação, a Lei de Informática e a própria legislação catarinense de Inovação, demonstram o potencial de desenvolvimento para esse setor (SEBRAE, 2010). Outro setor relevante para o crescimento dos esforços em inovação em Santa Catarina é o das indústrias de transformação, o qual possuía em 2011 mais de nove mil empresas, sendo responsável por mais de 168.000 empregos formais (SEBRAE, 2013).

\subsection{Métodos, técnicas e ferramentas para inovação}

Os Métodos, Técnicas e Ferramentas para Inovação podem ser definidos como os meios estruturados para aumentar a competitividade que podem suportar o processo de inovação nas empresas ajudando-as de forma sistemática a identificar e resolver problemas atendendo aos novos desafios do mercado (Phaal et al., 2006; Hidalgo; Albors, 2008; Igartua et al., 2010; D'Alvano; Hidalgo, 2012). A adoção de MTF-I, segundo a literatura, possui impacto positivo nas organizações durante o processo de desenvolvimento de novos produtos fazendo com que esse novo produto tenha sucesso no mercado (González; Palacios, 2002; Yeh et al., 2010; Graner; Mibler-Behr, 2013).

Ao longo dos anos, diferentes autores têm defendido a importância de uma cultura que suporta uma utilização cada vez maior de MTF-I (Alavi; Leidner, 2001; Vaccaro et al., 2010), pois eles representam um importante meio para melhorar as saídas do processo de desenvolvimento de novos produtos (Nijssen; Frambach, 2000) e possuem o objetivo de evitar o fracasso do projeto aumentando suas chances de sucesso (Nijssen; Lieshout, 1995). Eles ainda podem ser utilizados como facilitadores para apoiar a tomada de decisão e codificar o conhecimento e a aprendizagem (Scozzi et al., 2005).

A maior parte dos MTF-I são desenvolvidos para tratar de problemas específicos no processo de desenvolvimento de novos produtos. Dessa forma, sua utilização é destinada a fases específicas desse processo (Mahajan; Wind, 1992; Nijssen; Frambach, 2000; Chai; Xin, 2006). Por outro lado, os trabalhos relacionados ao tema apontam que eles também são úteis para atingir diversos objetivos e serem utilizados em diferentes estágios do processo de inovação (por exemplo, brainstorming e QFD) (Nijssen; Lieshout, 1995; Nijssen; Frambach, 2000; Chai; Xin, 2006).

Um dos principais motivos para a utilização de MTF-I, segundo Nijssen e Lieshout (1995), é a identificação de problemas e que esses motivos são modificados conforme se avança no processo de inovação. Os autores afirmam que nas fases iniciais do processo, a principal razão de se utilizar um MTF-I é identificar problemas, seguida de melhorar a taxa de sucesso do produto (Mahajan; Wind, 1992; Nijssen; Lieshout, 1995). Já ao final da fase de desenvolvimento, os principais motivos são fornecer suporte para a força de vendas e auxiliar no marketing do novo produto (Nijssen; Lieshout, 1995).

Outros benefícios da adoção de MTF-I são apontados pela literatura como qualidade do produto (Araújo et al., 1996), lucro bruto em relação aos concorrentes (Nijssen; Lieshout, 1995), identificação de requisitos do consumidor (Engelbrektsson; Soderman, 2004; Creusen et al., 2013), redução de tempo de desenvolvimento (Llorente Galera, 2009; Vaccaro et al., 2010), redução dos custos de desenvolvimento (Llorente Galera, 2009), e maturidade do processo de inovação (D'Alvano; Hidalgo, 2012).

Evidências empíricas sugerem que a maioria dos usuários de MTF-I estão satisfeitos com a performance deles (Mahajan; Wind, 1992; Nijssen; Lieshout, 1995; Nijssen; Frambach, 2000) e que esses são mais suscetíveis a experimentarem outros MTF-I (Nijssen; Frambach, 2000). Algumas dificuldades, tratadas como deficiências dos MTF-Is, são identificadas. Segundo Hidalgo e Albors (2008), elas parecem girar em torno da introdução do MTF-I na organização, pois isso significa um esforço extra que requer tempo, motivação e dinheiro. Nesse contexto, alguns estudos levantaram as principais dificuldades dos MTF-I, como por exemplo: complexidade do MTF-l; dificuldade de aprender (Mahajan; Wind, 1992; Chai; Xin, 2006); alto custo de implementação (Mahajan; Wind, 
1992); dificuldade de implementação (Nijssen; Lieshout, 1995); muito tempo para implementação; (Mahajan; Wind, 1992; Nijssen; Lieshout, 1995); e imprecisão de previsões (Nijssen; Frambach, 1998; Chai; Xin, 2006), entre outras.

Tendo em vista as dificuldades envolvidas na implementação e/ou utilização de um MTF-I, surge o desafio de motivar o apoio à gestão, de pensar no futuro e fomentar a criatividade, para instalar uma cultura e formulação de uma estratégia de inovação e para a implementação do processo de inovação (Hidalgo; Albors, 2008).

\section{PROCEDIMENTOS METODOLÓGICOS}

De forma a alcançar o objetivo desta pesquisa, inicialmente foi realizada uma revisão de literatura para aprofundar o contato com o tema, MTF-I, constatando-se a necessidade de mais estudos empíricos, uma vez que foram identificados poucos trabalhos dessa ordem. Para isso, utilizou-se a abordagem quantitativa de pesquisa, ou seja, aquela que considera que tudo pode ser quantificável, interpretando opiniões e informações a partir de medidas para classificá-las e analisá-las. Essa abordagem necessita da utilização de recursos estatísticos, como frequências, médias e outros (Moresi, 2004). Além de quantitativa, esta pesquisa também é considerada descritiva devido à natureza do relacionamento entre as variáveis pesquisadas, pois busca expor o fenômeno em estudo (Mattar, 2005).

Como estratégia de investigação para realização da pesquisa utilizou-se de um projeto de levantamento (survey). Segundo Babbie (2014), tal método consiste na aplicação de questionários a uma amostra da população alvo da pesquisa. Considerou-se essa a estratégia mais adequada à pesquisa uma vez que um projeto de levantamento descritivo avalia características de uma população (Gray, 2012) e descreve quantitativamente as suas tendências, atitudes ou opiniões a partir de uma determinada amostra (Mattar, 2005; Creswell, 2010; Fowler, 2014), ou seja, busca avaliar "o que" ocorreu, em vez de "porque" (Gray, 2012).

Como procedimento de coleta de dados, foi utilizado um levantamento (survey) pela internet. Em relação à coleta e à análise dos dados, estas foram realizadas em nível de projetos, pois uma mesma empresa pode ter um ou mais projetos de inovação em seu portfólio. Essa estratégia possui duas vantagens que merecem destaque. A primeira diz respeito ao fato de possibilitar um maior número de respondentes. A segunda está relacionada ao fato de que para projetos diferentes em uma mesma empresa, algumas das variáveis podem também ser diferentes.

A população alvo deste trabalho é formada por indivíduos que trabalharam ou trabalham com projetos de inovação, seja em produtos, processos, métodos de marketing ou métodos organizacionais, em empresas catarinenses. Para selecionar os elementos da amostra, utilizou-se o procedimento de amostragem não aleatória por conveniência, utilizando-se listas de contatos de empresas catarinenses disponibilizadas estritamente para o fim desta pesquisa.

Nesse sentido, enviou-se o questionário via correio eletrônico para um total de 220 pessoas, obtendo um retorno de 46, sendo esta a amostra deste estudo. Cabe salientar que, além dessas, foram obtidas 45 respostas incompletas, representando assim, um fator limitante do trabalho, uma vez que, se respondidos, traria uma amostra significativa à pesquisa. Meterko et al. (2015) destacam que apesar da baixa taxa de resposta, os resultados de estudos dessa natureza devem ser considerados, pois podem representar as atitudes da população em estudo.

Quanto ao instrumento de coleta de dados, este foi construído com 24 perguntas em que buscou-se identificar elementos como: setor e tamanho da organização, verificar a importância da inovação para a organização, se ela realiza inovações e quais motivos a levam a inovar; verificar quais MTI-I, os respondentes conhecem; buscar informações sobre os projetos de inovação da organização. Além disso, identificar os MTF-I utilizados no projeto. Por fim, indaga-se sobre a satisfação em relação ao MTF-I, identifica-se os benefícios e as deficiências e se o MTF-I continuou sendo utilizado após o projeto, bem como os dados dos respondentes. Com os dados coletados, inicialmente foram verificadas todas as respostas em termos de consistência, clareza e limpeza dos dados e, em seguida, utilizados softwares computacionais para organização dos dados e viabilizar as análises. 


\section{ANÁLISE E DISCUSSÃO DOS RESULTADOS}

Para responder a pergunta da pesquisa, inicialmente mapeou-se as características da inovação nas organizações catarinenses. Em seguida, identificou-se os MTF-I conhecidos e utilizados por elas, levantou-se os resultados dos projetos de inovação, e por fim, analisou-se a satisfação em relação à utilização dos Métodos, Técnicas e Ferramentas para Inovação nos projetos de inovação desenvolvidos pelas organizações catarinenses.

\subsection{Características da adoção de MTF-I no contexto catarinense}

De acordo com a Classificação Nacional de Atividades Econômicas (CNAE), as organizações podem ser classificadas em 21 setores da economia. Nesse sentido, entre as 46 respondentes, destaca-se os setores de Informação e Comunicação (13 respostas), Indústria de Transformação (12), e Educação (7). Ainda segundo o CNAE, cada setor é subdividido em setores específicos. Dessa forma, no setor de Informação e Comunicação, seis $(46,15 \%)$ organizações pertencem às atividades de serviços de tecnologia da informação, quatro $(30,77 \%)$ às atividades de prestação de serviços de informação, duas (15,38\%) à edição e edição integrada à impressão, e uma $(7,69 \%)$ pertence às atividades de telecomunicações. Já no setor das Indústrias de Transformação, os setores específicos das empresas respondentes foram: Fabricação de máquinas e equipamentos (2), Fabricação de equipamentos de informática, produtos eletrônicos e ópticos (2), Fabricação de produtos de metal, exceto máquinas e equipamentos (2), Metalurgia (2), Fabricação de produtos têxteis (1), Fabricação de produtos de borracha e de material plástico (1), Fabricação de produtos de minerais não metálicos (1), e Fabricação de produtos diversos (1).

Quanto ao tamanho da empresa, de acordo com o critério do Sebrae relativo ao número de empregados, uma organização com até 19 empregados é considerada uma microempresa, com 20 a 99 empregados, pequena empresa, com 100 a 499, média empresa, e a partir de 500 empregados, considera-se uma empresa de grande porte. Dessa forma, entre as respondentes, 14 (30\%) possuem 500 empregados ou mais, 9 (20\%) possuem de 100 a 499 empregados, 8 (17\%) possuem de 20 a 49, 6 (13\%) de 1 até 9 e de 50 até 99 empregados, e finalmente 3 (7\%) possuem de 10 a 19 empregados. O faturamento também pode ser utilizado como critério para verificar o tamanho da organização. Tal critério foi estabelecido pelo Banco Nacional de Desenvolvimento (BNDES), e considera: microempresa aquela com faturamento até $\$ 2,4$ milhões; pequena empresa a que tiver faturamento de $R \$ 2,4$ milhões até $R \$ 16$ milhões; média empresa com faturamento de $R \$ 16$ milhões até $R \$ 90$ milhões; médiagrande empresa de $R \$ 90$ milhões até $R \$ 300$ milhões; e empresa de grande porte aquela cujo faturamento é maior que R\$ 300 milhões. Assim, a partir dos dados obtidos, é possível perceber que entre os 46 respondentes, 16 podem ser classificadas como microempresas de acordo com esse critério. Entretanto, verifica-se que onze delas (24\%) possuem faturamento maior que $\mathrm{R} \$ 300$ milhões, ou seja, organizações de grande porte.

Tendo em vista que os dois critérios apontaram essa diferença quanto à classificação do tamanho das organizações, os dados sobre faturamento foram cruzados com os dados da quantidade de empregados. Como resultado obteve-se que $23,9 \%$ (11) das respondentes são empresas de grande porte para ambos os critérios, uma vez que possuem ao mesmo tempo 500 ou mais empregados e faturamento maior que $R \$ 300$ milhões, enquanto que 13\% (6) são microempresas, pois possuem de 1 até 9 empregados e faturamento inferior a R\$ 2,4 milhões.

Em relação aos projetos de inovação pesquisados, $34,8 \%$ tiveram como resultado inovações em produtos e o mesmo percentual em inovações em processos. Além disso, verificou-se a importância dos projetos para as organizações. Nesse sentido, os dados apontam que 59\% (27) deles foram muito importantes, enquanto que $35 \%$ (16) foram medianamente importantes. O trabalho de Stamm (2003) esclarece as principais diferenças entre a inovação radical e a inovação incremental. Dessa forma, o autor salienta que as inovações incrementais possuem projetos de curto prazo, em geral, de até dois anos de duração. Já as radicais possuem prazos maiores. Assim, é possível perceber que os projetos de inovação, de acordo com os pesquisados, tiveram curta duração, ou seja, até 1 ano (58,7\%), assim como os analisados por Graner e Mibler-Behr (2013). Parte expressiva também foi realizada entre 1 e 2 anos (28,3\%).

Nesse contexto, supõe-se que a maior parte dos projetos de inovação aqui analisados possuam foco em inovações incrementais, visto as diferenças identificadas por Stamm (2003). Destaca-se, também, o número de projetos realizados recentemente, sendo 26\% (12) em 2013 e 22\% (10) em 2015. 
No que diz respeito à visão sobre inovação, para $30(65,2 \%)$ dos 46 respondentes, a inovação é muito importante em seus negócios, seguido de $12(26,1 \%)$ respondentes afirmando que esse processo é medianamente importante. Além disso, chama atenção que $8,7 \%$ dos respondentes afirmaram que o processo de inovação é pouco importante para seus negócios. Dada essa importância do processo de inovação no contexto estudado, verificou-se a importância de cada tipo de inovação para os negócios das organizações. Em relação à inovação em produtos, $58,7 \%$ dos respondentes a consideram muito importante, e $23,9 \%$ medianamente importante. Da mesma forma, 60,9\% também considera a inovação em processos muito importante. Já a inovação em métodos de marketing e em métodos organizacionais são consideradas menos importante que as outras, uma vez que na primeira não houve maioria, ou seja, $34,8 \%$ delas a considera muito importante e o mesmo percentual medianamente importante. Por sua vez, a inovação em métodos organizacionais, segundo os respondentes (39,1\%), é medianamente importante para seus negócios, sendo muito importante para $37 \%$ delas.

Além da importância dada a cada tipo de inovação, verificou-se se as organizações as realizam em seus projetos, já que podem considerar importante, porém não as coloca em prática. Dessa forma, de acordo com os respondentes, $37 \%$ apenas realizam inovação em produtos, enquanto que $35 \%$ a realizam fortemente. Do mesmo modo, $37 \%$ realizam inovação em processos, $33 \%$ realizam fortemente, $24 \%$ realizam fracamente e apenas $3 \%$ não realizam. Quanto à inovação em métodos de marketing, observa-se que $33 \%$ a realizam, mas que $26 \%$ a realizam fracamente, seguido de $22 \%$ que não realizam e apenas $20 \%$ que a realizam fortemente. $O$ panorama da inovação em métodos organizacionais é semelhante quando se observa que $39 \%$ dos pesquisados afirmam que realizam esse tipo de inovação, enquanto que $33 \%$ delas a realizam fracamente.

Quanto aos motivos que levam as organizações a inovarem, de acordo com os pesquisados, os mais frequentes são: produzir com menores custos $(20,9 \%)$, gestão eficiente $(14,0 \%)$, e incrementar a penetração de mercado $(12,4 \%)$. Segundo os respondentes, os projetos analisados utilizaram certos critérios para mensurar a inovação. Nesse sentido, os mais utilizados foram o grau de satisfação do consumidor (11,4\%), e a produtividade $(11,4 \%)$, seguidos de volume de vendas $(9,8 \%)$, e utilização efetiva dos recursos $(8,9 \%)$. Diante disso, verifica-se congruência com os resultados do estudo realizado nos Estados Unidos por Mahajan e Wind (1992), o qual também apontou que os principais critérios utilizados para mensurar a inovação foram respectivamente a produtividade e o volume de vendas.

Quando se trata do nível de conhecimento sobre Métodos, Técnicas e Ferramentas para Inovação, é possível perceber que, de forma geral, as organizações conhecem muitos MTF-I. A partir de 572 citações, verificouse que o brainstorming $(91,30 \%)$ é o mais conhecido, seguido do benchmarking $(78,26 \%)$, da internet e intranets $(65,22 \%)$, da análise SWOT (63,04\%), e da pesquisa de mercado (63,04\%). Além dos disponíveis no instrumento de coleta de dados, foram citados outros nove MTF-I: análise da experiência do consumidor; análise de gaps; árvore do tema; árvore dos objetivos; balanced score card; explorando o benefício; KAIZEN; lentes da inovação; e world café.

Verificou-se, a partir dos dados levantados, que em média são conhecidos 13 MTF-I por respondente e que pode existir uma correlação positiva entre o nível de importância dada à inovação e à quantidade de Métodos, Técnicas e Ferramentas para Inovação conhecidos. Assim, tal questão pode ser investigada a fundo por outros estudos para verificar a relação entre essas variáveis. O conhecimento sobre tais MTF-I não representa sua adoção. Por isso, questionou-se quais MTF-I efetivamente são adotados e utilizados pela organização respondente. Dessa forma, percebeu-se que os mais conhecidos não são os mais utilizados, exceto o brainstorming e o benchmarking que aparecem no topo da lista de ambos. Entre os mais adotados, segundo os respondentes, seguidos do brainstorming $(71,74 \%)$ e do benchmarking (26,09\%), estão a análise de viabilidade $(21,74 \%)$, a análise de cenários $(15,22 \%)$, e o teste de uso em casa $(15,22 \%)$.

A partir do agrupamento de todos os dados relacionados aos MTF-I nessa pesquisa foi possível obter uma visão geral acerca do tema proposto, fornecendo assim um panorama do contexto catarinense em relação à adoção de Métodos, Técnicas e Ferramentas para Inovação. Dessa forma, verificou-se que a maior parte é muito utilizada principalmente no front end da inovação, mas que também são bastante considerados quando se trata das fases de desenvolvimento e implementação.

Gerar novas ideias (46 citações), identificar problemas ( 28 citações) e os requisitos dos consumidores ( 28 citações) foram os motivos que mais levaram as organizações pesquisadas a adotarem MTF-I em seus processos de inovação. De maneira geral, há um alto grau de satisfação apontado pelos adotantes de MTF-l, sendo que evidências empíricas já sugeriam esse fato (Mahajan; Wind, 1992; Nijssen; Lieshout, 1995; Nijssen; Frambach, 
2000). Dessa forma $91,97 \%$ dos MTF-I foram utilizados novamente em outros projetos. A maior parte daqueles que não foram utilizados, segundo os respondentes, foi devido à não participação em outros projetos.

Por fim, indagou-se quanto aos benefícios e as deficiências dos MTF-I. Nesse sentido, identificou-se que os benefícios mais citados foram a melhoria da taxa de sucesso do produto, captura de informações e identificação de problemas. Já as maiores deficiências, percebeu-se que, de acordo com os dados obtidos, o tempo para implementação e a previsibilidade podem apresentar dificuldades dependendo do MTF-I utilizado.

\section{CONSIDERAÇÕES FINAIS}

Este estudo teve como objetivo descrever as principais características da adoção de Métodos, Técnicas e Ferramentas para Inovação em organizações catarinenses. Nesse sentido, inicialmente, realizou-se um levantamento em 46 organizações localizadas no Estado de Santa Catarina para identificar essas características e ao final, confrontou-se os resultados obtidos com a literatura sobre o tema para dar suporte a esses achados e resposta a pergunta da pesquisa.

Os principais resultados em relação à inovação demonstraram que 11 das 46 respondentes são grandes empresas ao se considerar tanto o critério faturamento quanto o número de empregados. Essas empresas, segundo os resultados, consideram a inovação um processo muito importante para seus negócios. Quanto aos resultados do processo de inovação, assim como na literatura, ainda se verifica bastante foco em produto e em processos.

Quanto ao conhecimento acerca de MTF-I, os resultados apontaram que as organizações conhecem muitos (em média 13), porém quando se trata em adotá-los esse número fica bastante reduzido, ou seja, próximo a três por organização. No que tange aos projetos de inovação, verificou-se que eles são importantes para as organizações e que são realizados por curto prazo (até dois anos).

Os MTF-I mais utilizados pelas organizações catarinenses pesquisadas foram respectivamente o brainstorming, o benchmarking e a análise de viabilidade. De maneira geral, os MTF-I são utilizados com maior ênfase no front end da inovação, mas que são utilizados também nas fases de desenvolvimento e de implementação, demonstrando a possibilidade de utilização de forma flexível e criativa para atenderem aos objetivos dos projetos.

Segundo as organizações pesquisadas, os maiores motivos para adotar MTF-I foram gerar novas ideias, identificar requisitos do consumidor e resolver problemas. Diante disso, os MTF-I parecem atender aos objetivos que são propostos quando adotados, pois percebeu-se grande satisfação após sua utilização. Os benefícios e as deficiências dos MTF-I também foram questionados, e, em geral, os resultados indicam que os maiores benefícios parecem ser a melhoria da taxa de sucesso do produto, a captura de informações e a identificação de problemas. Já as deficiências, foram o tempo de implementação e a dificuldade de previsibilidade dos MTF-I.

Esta pesquisa contribui empiricamente fornecendo uma descrição das características da adoção de Métodos, Técnicas e Ferramentas para Inovação em organizações catarinenses. Visto que esse é um tema emergente, necessita-se ainda de maiores aprofundamentos para elucidar diversas lacunas apontadas tanto pela literatura quanto neste trabalho. Nesse contexto, outras pesquisas poderão, por exemplo, ampliar o presente levantamento para o contexto brasileiro, utilizando-se procedimentos de amostragem para que os resultados sejam representativos, e assim, generalizá-los.

Como principais limitações do trabalho, destaca-se aspectos como a impossibilidade de generalização dos resultados, baixo interesse dos respondentes em participar da pesquisa, e falta de recursos humanos para coleta de dados, uma vez que identificou-se que outros estudos dessa natureza utilizaram profissionais de telesserviços.

Diante do exposto, a inovação ainda pode representar um desafio para as organizações. Assim, novas estratégias devem emergir para estimular essa cultura para inovação. Nesse sentido, essa pesquisa indica que os Métodos, Técnicas e Ferramentas para Inovação parecem ser um meio realmente efetivo para alcançar os objetivos estratégicos das organizações. 


\section{REFERÊNCIAS}

Alavi, M., \& Leidner, D. (2001). Review: Knowledge management and knowledge management systems: conceptual foundations and research issues. MIS Quarterly, 25(1), 107-136.

Araujo, C. S., Benedettoneto, H., Campello, A. C., Segre, F. M., \& Wright, I. C. (1996). The utilization of product development methods: A survey of UK industry. Journal of Engineering Design, 7(3).

Babbie, Earl. (2014). The practive of social research. (14. ed.). Boston: Cengage Learning.

Baregheh, A., Rowley, J., \& Sambrook, S. (2009). Towards a multidisciplinary definition of innovation. Management Decision, 47(8).

BNDES. Recuperado de http://www.bndes.gov.br/SiteBNDES/bndes/bndes_pt/Institucional/Apoio_Financeiro/porte.html

Brady, T., Rush, H., Hobday, M., Davies, A., Probert, D., \& Banerjee, S. (1997). Tools for technology management: An academic perspective. Technovation, 17(8).

Buchele, G. T. (2015). Adoção de métodos, técnicas e ferramentas para inovação: Um levantamento em organizações catarinenses. (Dissertação de Mestrado, Universidade Federal de Santa Catarina). Recuperado de https://repositorio.ufsc.br/xmlui/handle/123456789/159034

Buchele, G. T., Teza, P., Dandolini, G. A., \& Souza, J. A. (2015). Análise dos artigos qualitativos empíricos sobre métodos, técnicas e ferramentas para inovação. Revista de Administração Mackenzie, 16(3).

Chai, K. H., \& Xin, Y. (2006). The application of new product development tools in industry: the case of Singapore. IEEE Transactions on Engineering Management, 53(4).

CNAES. Recuperado de http://www.cnae.ibge.gov.br/estrutura.asp?TabelaBusca=CNAE_200@CNAE\%202.1

Creswell, J. W. (2010). Projeto de pesquisa: Métodos qualitativo, quantitativo e misto. (3. ed.). Porto Alegre: Artmed.

Creusen, M., Hultink, E. J., \& Eling, K. (2013). Choice of consumer research methods in the front end of new product development. Int. Journal of Market Research, 55(1).

D'alvano, L., \& Hidalgo, A. (2012). Innovation management techniques and development degree of innovation process in service organizations. R\&D Management, 42(1).

Engelbrektsson, P., \& Soderman, M. (2004). The use and perception of methods and product representations in product development: A survey of Swedish industry. Journal of Engineering Design, 15(2).

Fowler, F. J. (2014). Survey research methods. (5. ed.). New York: SAGE Publications.

González, F. J. M., \& Palacios, T. M. B. (2002). The effect of new product development techniques on new product success in Spanish firms. Industrial Marketing Management, 31(3).

Graner, M., \& Mißler-Behr, M. (2013). Key determinants of the successful adoption of new product development methods. European Journal of Innovation Management, 16(3).

Gray, David E. (2012). Pesquisa no mundo real. (2. ed.). Porto Alegre: Penso.

Hidalgo, A., \& Albors, J. (2008). Innovation management techniques and tools: A review from theory and practice. $R$ and D Management, 38(2).

Igartua, J. I., Garrigós, J. A., \& Hervas-Oliver, J. L. (2010). How innovation management techniques support an open innovation strategy. Research Technology Management, 53(3).

Llorente Galera, F. (2009). Tecnological innovation, systems and techniques used in $r+d$ by catalonian direct suppliers of oems. Investigaciones Europeas de Direccion y Economia de la Empresa, 15(2).

Mahajan, V., \& Wind, J. (1992). New product models: Practice, shortcomings and desired improvements. The Journal of Product Innovation Management, 9(2).

Mattar, F. N. (2005). Pesquisa de marketing: Metodologia, planejamento. (6. ed.). São Paulo: Atlas.

Meterko, M., Restuccia, J. D., Stolzmann, K., Mohr, D., Brennan, C., Glasgow, J., \& Kaboli, P. (2015). Response rates, nonresponse bias, and data quality: Results from a national survey of senior healthcare leaders. Public Opinion Quarterly, 79(1).

Moresi, E. (2004). Metodologia da pesquisa. Brasília: UCB.

Nicholas, J., Ledwith, A., \& Bessant, J. (2015). Selecting early-stage ideas for radical innovation: Tools and structures. Research-Technology Management, 58(4).

Nijssen, E. J., \& Frambach, R. T. (2000). Determinants of the adoption of new product development tools by industrial firms. Industrial Marketing Management, 29(2).

Nijssen, E. J., \& Frambach, R. T. (1998). Market research companies and new product development tools. Journal of Product \& Brand Management, 7(4).

Nijssen, E. J., \& Lieshout, K. F. M. (1995). Awareness, use and effectiveness of models and methods for new product development. European Journal of Marketing, 29(10).

Phaal, R., Farrukh, C. J. P., \& Probert, D. R. (2006). Technology management tools: Concept, development and application. Technovation, 26(3).

Phaal, R., Kerr, C., Oughton, D., \& Probert, D. (2012). Towards a modular toolkit for strategic technology management. International Journal of Technology Intelligence and Planning, 8(2).

Ranking de Gestão e Competivividade dos Estados Brasileiros (2014). Recuperado de http://www.clp.org.br/2013/?thinktank=indicaores-dos-estados 
Scozzi, B., Garavelli, C., \& Crowston, K. (2005). Methods for modeling and supporting innovation processes in SMEs. European Journal of Innovation Management, 8(1).

SEBRAE. (2014). Coletânea de informações socioeconômicas de Santa Catarina. Florianópolis: Sebrae/SC.

SEBRAE. (2010). Santa Catarina em números: Tecnologia. Florianópolis: Sebrae/SC.

SEBRAE. (2013). Santa Catarina em números: Macrorregiões. Florianópolis: Sebrae/SC.

Shehabuddeen, N., Probert, D., Phaal, R., \& Platts, K. (1999). Representing and approaching complex management issues: Part 1 - Role and definition. Cambridge: Centre for Technology Management.

Stamm, B. V. (2003). Managing innovation, design \& creativity. Nova Jersey: Wiley.

Tian, X., \& Wang, T. Y. (2014). Tolerance for failure and corporate innovation. The Review of Financial Studies, 27(1).

Tidd, J., \& Thuriaux-Alemán, B. (2016). Innovation management practices: Cross-sectorial adoption, variation, and effectiveness. R\&D Management.

Vaccaro, A., Parente, R., \& Veloso, F. M. (2010). Knowledge management tools, inter-organizational relationships, innovation and firm performance. Technological Forecasting and Social Change, 77(7).

Vaseashta, A. (2014). Advanced sciences convergence based methods for surveillance of emerging trends in science, technology, and intelligence. Foresight, 16(1).

Yeh, T. M., Pai, F. Y., \& Yang, C. C. (2010). Performance improvement in new product development with effective tools and techniques adoption for high-tech industries. Quality and Quantity, 44(1). 